\title{
Morfología de nopal forrajero cv Miúda (Nopalea cochenillifera Salm Dyck) en sistemas de cultivo del agreste de Pernambuco, Brasil
}

Paulina Vazquez Mendoza ${ }^{a^{*}}$

Toni Carvalho de Sousa ${ }^{\mathrm{b}}$

Mercia Virginia Ferreira Dos Santos ${ }^{b}$

Oscar Vicente Vazquez Mendoza ${ }^{c}$

Jose Carlos Batista Dubeux Junior ${ }^{d}$

Mario de Andrade Lira ${ }^{b}$

${ }^{a}$ Universidad Autónoma de Guerrero. Centro Regional de Educación Superior de la Costa Chica. 41800, Florencio Villarreal, Guerrero. México.

${ }^{\mathrm{b}}$ Universidade Federal Rural de Pernambuco. Departamento de Zootecnia. Pernambuco. Brasil.

${ }^{\mathrm{c}}$ NOREL México SA DE CV, Querétaro, México

${ }^{\mathrm{d}}$ University of Florida. North Florida Research and Education Center, Marianna, Florida, USA.

* Autor de correspondencia: vazmepa@gmail.com 


\section{Resumen:}

Las características morfológicas de las plantas forrajeras como el nopal, son influenciadas de acuerdo al manejo recibido, prácticas como la fertilización son importantes; debido a lo anterior el objetivo de esta investigación fue evaluar el efecto de la fertilización con materia orgánica (MO) $\left(0,10,000,20,000\right.$ y 30,000 kg MO ha-1 año ${ }^{-1}$ con estiércol bovino) o mineral $\left(0,120,240\right.$ y $360 \mathrm{~kg}$ de $\mathrm{N} \mathrm{ha}^{-1}$ año $^{-1}$ utilizando urea) y la frecuencia de corte (anual y bianual) en la longitud, ancho, perímetro, e Índice del Área de Cladodio (IAC) en cladodios de nopal forrajero cv. Miúda (Nopalea cochenillifera Salm Dyck), y su relación con la productividad. El diseño experimental fue de bloques al azar, con el arreglo de parcelas sub-sub-divididas con cuatro repeticiones. La fertilización con 30,000 $\mathrm{kg} \mathrm{MO} \mathrm{ha}^{-1} \mathrm{año}^{-1}$ incrementó el ancho y longitud de cladodio de 9.8 a $17.8 \%$ respecto al tratamiento testigo. El perímetro de cladodio se incrementó proporcionalmente cuando aumentó la MO. El IAC fue $68.29 \%$ mayor (con $25970 \mathrm{~kg} \mathrm{ha}^{-1}$ ) respecto al tratamiento testigo. La fertilización mineral sólo afectó el perímetro de cladodio en el corte anual con dosis de $120 \mathrm{~kg} \mathrm{ha}^{-1} \mathrm{y}$ el IAC fue mayor en el corte bianual. Se concluyó que la fertilización con MO aumentó el ancho, longitud e IAC en cladodios de nopal forrajero cv Miúda (Nopalea cochenillifera Salm Dyck), mientras que la fertilización mineral tuvo bajo impacto en el ancho, longitud e IAC, el corte bianual favorece el IAC. Se presentó una alta correlación entre las variables evaluadas y la producción de materia seca.

Palabras clave: Nopal forrajero, Cladodio (IAC), Fertilización, Frecuencia de corte.

Recibido: 24/02/2017

Aceptado: 22/08/2018

La región noreste de Brasil representa $18.27 \%$ del territorio nacional, del cual $62.11 \%$ es semiárido $^{(1)}$. En esta región, la distribución anual de lluvias $(500 \mathrm{~mm})^{(2)}$ es irregular lo cual causa una fuerte escasez de forraje para la alimentación de rumiantes. Las especies de nopal Opuntia sp. y Nopalea sp. están adaptadas a condiciones de escasez de agua, altas temperaturas y suelos pobres ${ }^{(3,4,5)}$, por lo cual son una alternativa valiosa para alimentación de los animales, especialmente en el período de secas. Opuntia ficus-indica Mill y Nopalea cochenillifera Salm Dyck son los dos géneros de nopal con mayor cultivo en el noreste brasileño $^{(6)}$.

El nopal forrajero cv. Miúda (Nopalea cochenillifera Salm Dyck) también conocido como nopal Dulce, tiene la ventaja de ser resistente a la grana cochinilla (Dactylopius opuntiae Cockerell $)^{(7,8)}$. Esta característica, aunada a su contenido de PC de $6.2 \%$, FDN de $26 \%$ y digestibilidad de $78 \%{ }^{(9)}$, lo hace una planta con uso potencial para la alimentación del 
ganado, aunque su cultivo al igual que otros requiere una adecuada fertilidad del suelo. La fertilización orgánica y mineral en el manejo del cultivo de nopal es una práctica común y se emplea para compensar la extracción de nutrientes del suelo por el cultivo además es una estrategia importante para aumentar la eficiencia de la producción de forraje ${ }^{(10,11)}$.

Las variables usadas en estudios de ecofisiología de las plantas forrajeras muestran respuestas diferentes según el manejo de la planta; en el caso de las características morfológicas del cladodio, éstas muestran una relación directa con el rendimiento en materia verde y seca del nopal $^{(12)}$. Sin embargo, existen pocos estudios sobre las características morfológicas como indicador de la productividad. Por lo tanto, el objetivo de esta investigación fue evaluar el efecto de la fertilización orgánica y mineral, así como la frecuencia de corte de cladodios sobre las características morfológicas del nopal forrajero cv Miúda (Nopalea cochenillifera Salm Dyck) cultivado en el Agreste de Pernambuco, Brasil.

El experimento se realizó entre junio de 2011 y mayo de 2013, en la Estación Experimental del Instituto Agronómico de Pernambuco, municipio de Caruaru, en la región Agreste de Pernambuco, zona de transición entre la zona de bosque tropical húmedo y el semiárido. Esta región posee un suelo pedregoso, vegetación escasa $(<40$ y $>20 \%$ de cobertura) y de tamaño pequeño $(<1.5 \mathrm{~m} \text { altura })^{(13)}$. Se ubica en el noreste de Brasil, a $8^{\circ} 14^{\prime} \mathrm{S}$ y $35^{\circ} 55^{\prime} \mathrm{O}$, a una altitud de 575 msnm y en suelo Neossolo Regolítico ${ }^{(14)}$. Durante el periodo del experimento, la precipitación en el lugar fue de $1,068.3 \mathrm{~mm}$ y su variación durante este periodo se muestra en la Figura 1.

Figura 1: Precipitación $(\mathrm{mm})$ mensual y temperaturas máxima y mínima en Caruaru, Agreste de Pernambuco, de enero 2011 a junio 2013

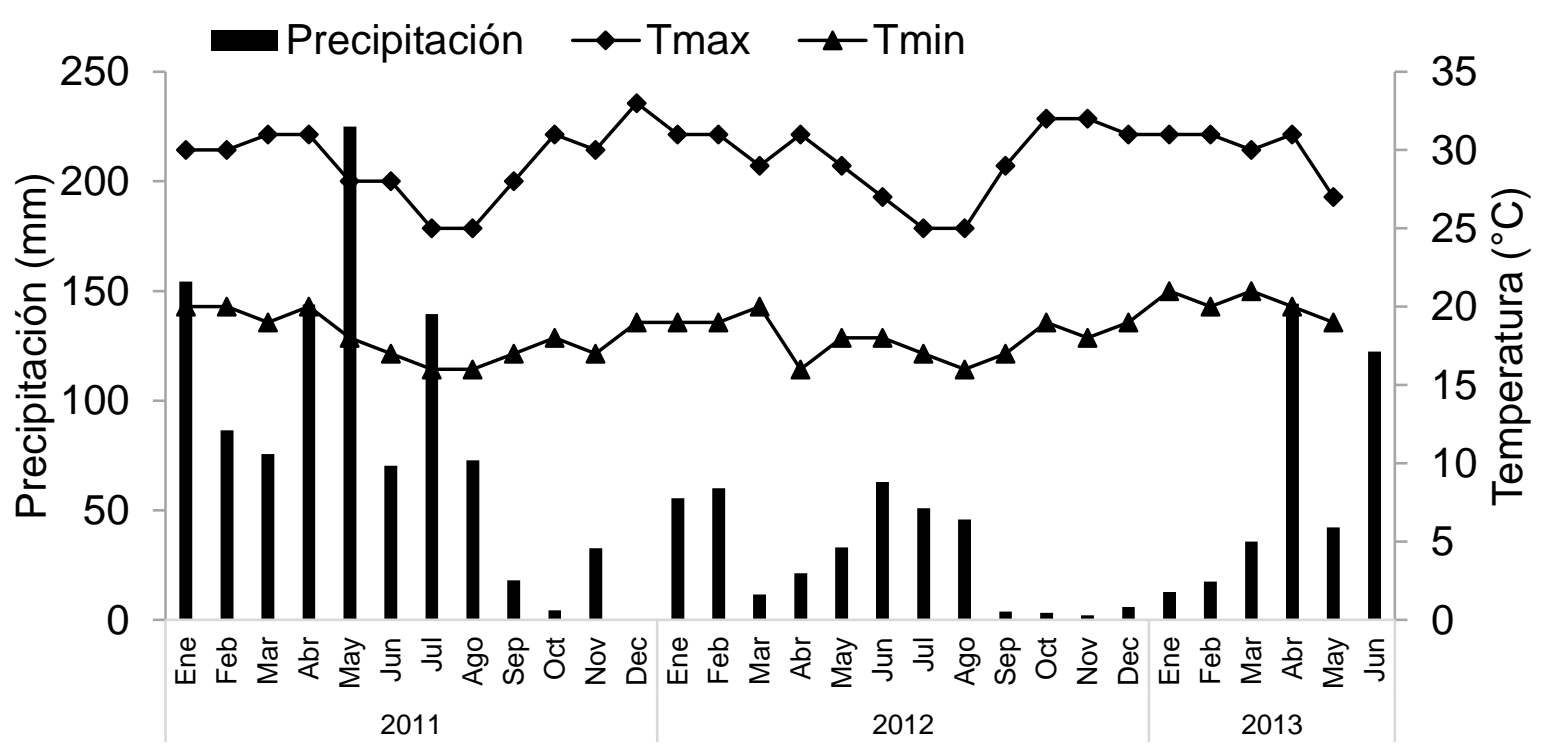


Para el experimento se tomaron muestras de suelo de entre 0 y $20 \mathrm{~cm}$ de profundidad y se determinaron sus características químicas usando el método de análisis de suelo de la Empresa Brasileira de Pesquisa Agropecuária ${ }^{(15)}$ (Cuadro 1).

Cuadro 1: Características químicas del suelo del área experimental en Caruaru Agreste de Pernambuco, Brasil; antes de la fertilización

\begin{tabular}{|c|c|c|c|c|c|}
\hline Componente & Media & EEM & Componente & Media & EEM $^{\text {IIIII }}$ \\
\hline $\mathrm{pH}$ (agua) & 4.78 & 0.1 & Sodio, $\mathrm{mg} \mathrm{dm}^{-3}$ & 11.50 & 0.01 \\
\hline Fósforo, $\mathrm{mg} \mathrm{dm}^{-3 \dagger}$ & 10.45 & 3.81 & Aluminio, $\mathrm{mg} \mathrm{dm}^{-3}$ & 17.98 & 0.03 \\
\hline Potasio, $\mathrm{mg} \mathrm{dm}^{-3}$ & 74.29 & 0.04 & Hidrógeno, $\mathrm{mg} \mathrm{dm}^{-3}$ & 24.70 & 0.14 \\
\hline Calcio, $\mathrm{mg} \mathrm{dm}^{-3}$ & 428.00 & 0.26 & S.B. ${ }^{\text {II }}, \mathrm{cmolc} \mathrm{dm}^{-3}$ & 2.78 & 0.33 \\
\hline Magnesio, $\mathrm{mg} \mathrm{dm}^{-3}$ & 48.62 & 0.05 & $\mathrm{CTC}^{\S}, \mathrm{cmolc} \mathrm{dm}^{-3}$ & 5.46 & 0.38 \\
\hline Manganeso, $\mathrm{mg} \mathrm{dm}^{-3}$ & 70.42 & 10.27 & $\mathrm{~V}^{\mathrm{p}}, \%$ & 50.05 & 3.21 \\
\hline Zinc, $\mathrm{mg} \mathrm{dm}^{-3}$ & 12.46 & 1.66 & Carbono, $\%$ & 1.15 & 0.06 \\
\hline Fierro, $\mathrm{mg} \mathrm{dm}^{-3}$ & 46.20 & 3.06 & $\mathrm{M}^{\mathrm{a}}, \%$ & 8.15 & 1.97 \\
\hline Cobre, $\mathrm{mg} \mathrm{dm}^{-3}$ & 0.06 & 0.02 & $\mathrm{MO}^{\dagger \dagger}, \%$ & 1.97 & 0.10 \\
\hline
\end{tabular}

${ }^{\dagger}$ Mehlich 1; "Isuma de bases; ${ }^{\S}$ capacidad de intercambio catiónico; ${ }^{\mathrm{p}}$ saturación por bases; ${ }^{\text {a }}$ saturación por aluminio; ${ }^{\dagger}$ materia orgánica del suelo; ${ }^{\mathbb{T U}}$ error estándar de la media.

Los tratamientos fueron: fertilización orgánica con 0, 10,000, 20,000 y 30,000 kg de MO ha ${ }^{1}$ año $^{-1}$ de estiércol bovino; fertilización mineral con 0, 120, 240 y $360 \mathrm{~kg} \mathrm{~N} \mathrm{ha}^{-1} \mathrm{año}^{-1}$, con urea; y dos frecuencias de corte (anual y bianual). El diseño experimental fue de bloques al azar con un arreglo en parcelas sub-sub-divididas, con cuatro repeticiones. La parcela mayor $(14.4 \times 8.0 \mathrm{~m})$ se usó para probar los niveles de materia orgánica; las subparcelas $(7.2 \times 8.0$ $\mathrm{m}$ ), para evaluar las frecuencias de corte, y la sub-sub-parcela $(14.4 \times 2.0)$, para evaluar los niveles de nitrógeno. La unidad experimental se formó por seis hileras de plantas. Las dos hileras laterales y tres plantas por extremo se consideraron como bordes. El área efectiva de muestreo fue de $33.84 \mathrm{~m}^{2}$ con 282 plantas.

La siembra se realizó entre abril y mayo de 2011. Cladodios maduros del nopal forrajero se sembraron en línea con separación de $1.2 \mathrm{~m}$ entre líneas y de $0.1 \mathrm{~m}$ entre cladodios. La densidad fue de 83,336 plantas por hectárea. La fertilización orgánica se hizo al momento de la siembra (junio de 2011) y después del primer corte anual (junio de 2012). El estiércol contenía $1.1,3.74$ y $16.5 \mathrm{~g} \mathrm{~kg}^{-1}$ de $\mathrm{N}$, P y K según determinaciones de la $\mathrm{AOAC}^{(16)}$. La fertilización mineral en el primer año de cultivo se realizó entre el 5 de junio y 19 julio de 2011, mientras que la segunda fue el 28 de junio, 23 de julio y 19 de agosto de 2012. La cosecha de cladodios fue completa y se conservó únicamente la planta madre. La medición de longitud $(\mathrm{cm})$, ancho $(\mathrm{cm})$ y perímetro $(\mathrm{cm})$ de cladodio, se delineó en papel A4, el contorno de los cladodios de dos plantas por subsubparcela, y se examinaron con el Analizador de Área foliar (Portable Laser Leaf Area Meter CI -202 Bio-Science Inc.®). 
También se evaluó el IAC al sumar el área de los cladodios de la planta $\left(\mathrm{m}^{2}\right)$ y dividirla entre la superficie del suelo $\left(0.12 \mathrm{~m}^{2}\right)$ ocupada por cada planta; en este caso se consideraron ambos lados de los cladodios ${ }^{(17)}$, se determinó el coeficiente de correlación entre producción de $\mathrm{MS}^{(18)}$ y las variables, longitud, ancho, perímetro e IAC . Los datos se analizaron con el procedimiento MIXTO de SAS $^{(19)}$. La prueba de Tukey $(P \leq 0.05)$ se usó para el factor frecuencia de corte, y contrastes ortogonales polinomiales $(P \leq 0.05)$ para los factores: fertilización orgánica y nitrogenada.

La longitud, el ancho y el IAC del nopal forrajero no mostraron efectos $(P>0.05)$ por la fertilización mineral debido, probablemente, a la irregularidad de la precipitación ocurrida durante el período experimental (Figura 1), lo cual afectaría la absorción de nutrientes o el bajo contenido de materia orgánica (Cuadro 1). Se han reportado efectos similares con la fertilización mineral, en las características morfológicas y la producción de biomasa en nopal forrajero cv. Miúda ( $N$. cochenillifera), los autores lo atribuyeron al sistema radical, cuyo crecimiento responde a la precipitación pluvial, por lo que la distribución irregular de las lluvias afecta la eficiencia de absorción de nitrógeno e incrementa la pérdida de nutrientes a través de lixiviación o volatilización cuando hay exceso o ausencia lluvia respectivamente ${ }^{(20)}$. Se han observado efectos positivos de la fertilización nitrogenada y fosfatada mineral, en la producción de otras especies de nopal como Opuntia lindheimeri sólo después de dos años de establecido el plantío ${ }^{(21)}$. La fertilización orgánica mejora el crecimiento y la producción de los cultivos $^{(22)}$. En el experimento, el ancho y la longitud de cladodio aumentaron proporcionalmente con la fertilización orgánica $\left(\mathrm{R}^{2}=0.26\right.$ y $\left.0.47 ; P \leq 0.001\right)$, y alcanzaron 9.8 y $17.8 \%$ de incremento respecto al testigo $(P \leq 0.05)$ cuando se fertilizó con $30,000 \mathrm{~kg} \mathrm{ha}^{-1}$ año ${ }^{-1}$ (Figura 2).

Figura 2: Longitud y ancho del cladodio de nopal forrajero cv. Miúda (Nopalea cochenillifera Salm Dyck) fertilizado con estiércol bovino (Agreste of Pernambuco, Brasil)

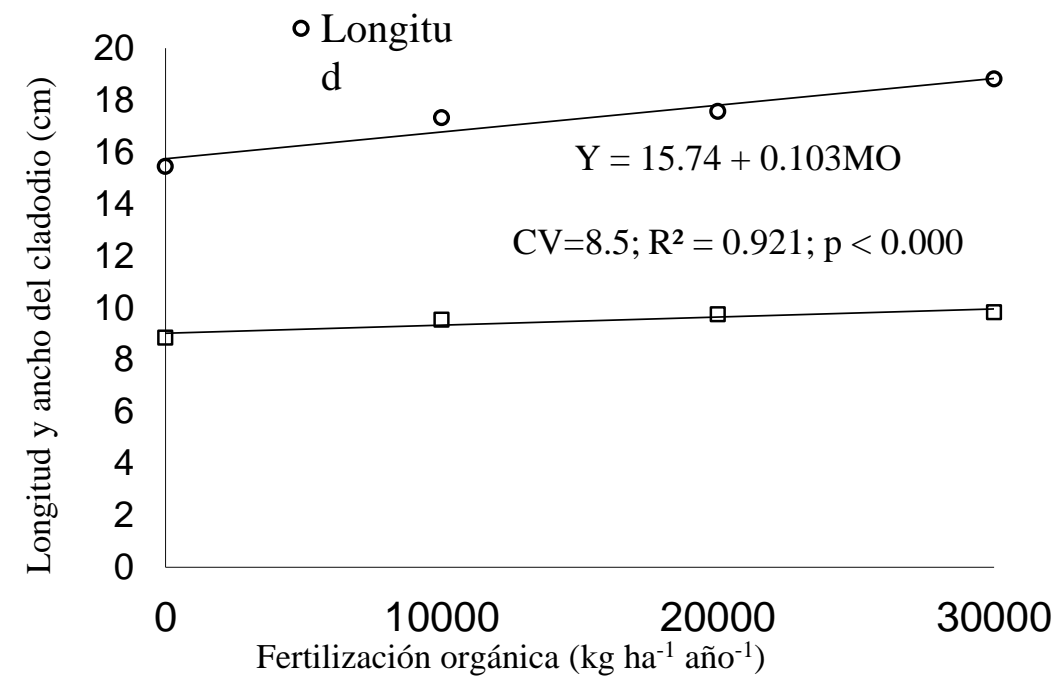


Estudios mostraron que la longitud del cladodio de $O$. ficus-indica cv Lisa forrajera aumentó gradualmente de acuerdo a la fertilización orgánica con estiércol bovino, en dosis de 20,000, 40,000 y $60,000 \mathrm{~kg} \mathrm{ha}^{-1} \mathrm{año}^{-1}$ y el testigo con 0 fertilización, durante tres años consecutivos $^{(23)}$. La longitud de cladodio en $O$. ficus-indica cv Gigante, de 600 días de edad y con aplicación de $90,000 \mathrm{~kg} \mathrm{ha}^{-1}$ año $^{-1}$ de estiércol bovino incrementó sólo $8 \%$ respecto al testigo $^{(24)}$.

Respecto a la anchura de cladodio en $O$. ficus-indica, no se encontró efecto con la aplicación de estiércol bovino $(P>0.05)$, en dosis de $0,20,000,40,000$ y 60,000 kg ha ${ }^{-1(23)}$. La fertilización orgánica, mineral y la frecuencia de corte anual afectaron el perímetro del cladodio $(P \leq 0.05)$. Este incremento fue linealmente mayor $(P \leq 0.05)$ conforme el nivel de materia orgánica aplicada al suelo. El incremento del perímetro de cladodio dependió de la interacción con la fertilización mineral y la frecuencia de corte (Figura 3). El perímetro de cladodio se incrementó a una tasa de $0.339 \mathrm{~cm}$ por cada $1,000 \mathrm{~kg}$ de $\mathrm{MO}$ y $360 \mathrm{~kg}$ de $\mathrm{N}$ por

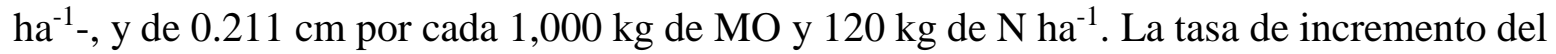
perímetro de cladodio fue mayor cuando se realizó el corte anual $(0.211 \mathrm{~cm}$ por cada 1,000 $\mathrm{kg}$ de MO) con respecto al bianual $(0.0304 \mathrm{~cm}$ por cada $1,000 \mathrm{~kg}$ de $\mathrm{MO})$.

Figura 3: Perímetro de cladodio de nopal forrajero cv. Miúda (Nopalea cochenillifera Salm Dyck) por efecto de la fertilización orgánica, mineral y frecuencia de corte (Agreste de Pernambuco, Brasil)

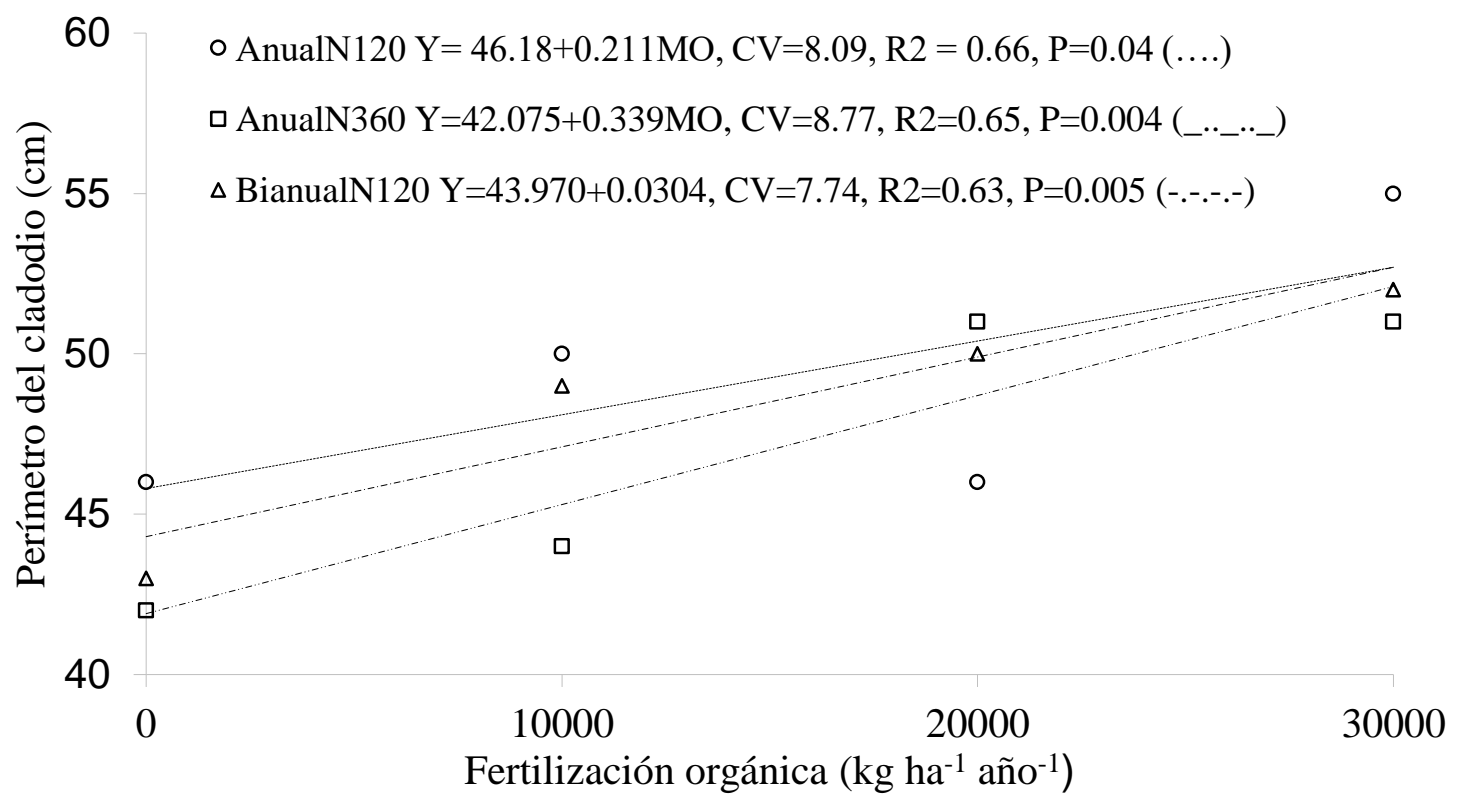


El efecto de sombreado es un factor importante que se presenta cuando se usan altas densidades de plantas; lo que compromete la exposición del área fotosintéticamente activa y origina con ello una menor producción ${ }^{(25)}$.

La Figura 4 muestra el efecto de la fertilización orgánica en el IAC, el cual mostró una tendencia cuadrática $(P \leq 0.05)$ en la que su aumento fue proporcional al aplicar 10,000 y $20,000 \mathrm{~kg}_{\text {de }} \mathrm{MO} \mathrm{ha}{ }^{-1} \mathrm{año}^{-1}$, para alcanzar un máximo IAC con 25,970 kg de MO ha ${ }^{-1} \mathrm{año}^{-1}$. Las dosis de fertilización orgánica mayores a $25,970 \mathrm{~kg} \mathrm{ha}^{-1} \mathrm{año}^{-1}$ no incrementaron el IAC. El espaciamiento entre plantas y la dosis de fertilización orgánica afectaron el IAC de Opuntia ficus-indica cv Gigante, estudios reportan mayores IAC con espaciamientos de $1 \mathrm{x} 0.5 \mathrm{~m}$ y con dosis de fertilización orgánica entre 60,000 y $90,000 \mathrm{~kg} \mathrm{ha}^{-1} \mathrm{año}^{-1(24)}$; valores de espaciamiento y fertilización superiores a los definidos en este estudio. Este comportamiento en el IAC se repite con otro tipo de abonos orgánicos, incrementos del $30 \%$ en el área fotosintéticamente activa de la planta se presentan con la aplicación de bioabono a base de guano y cladodios picados procedentes de la poda, aplicado a razón de $15,000,30,000,45,000$ y $60,000 \mathrm{~kg} \mathrm{ha}^{-1} \mathrm{año}^{-1(26)}$. El incremento de $30 \%$ en el IAC es menor a los reportados en el presente trabajo, ya que el IAC incrementó en $68.9 \%$ con la aplicación de $25,970 \mathrm{~kg} \mathrm{ha}^{-1}$ año $^{-1}$ de estiércol bovino, respecto al tratamiento testigo.

Figura 4: Índice de área de cladodio de nopal forrajero cv. Miúda (Nopalea cochenillifera Salm Dyck), por efecto de la fertilización orgánica (Agreste de Pernambuco, Brasil)

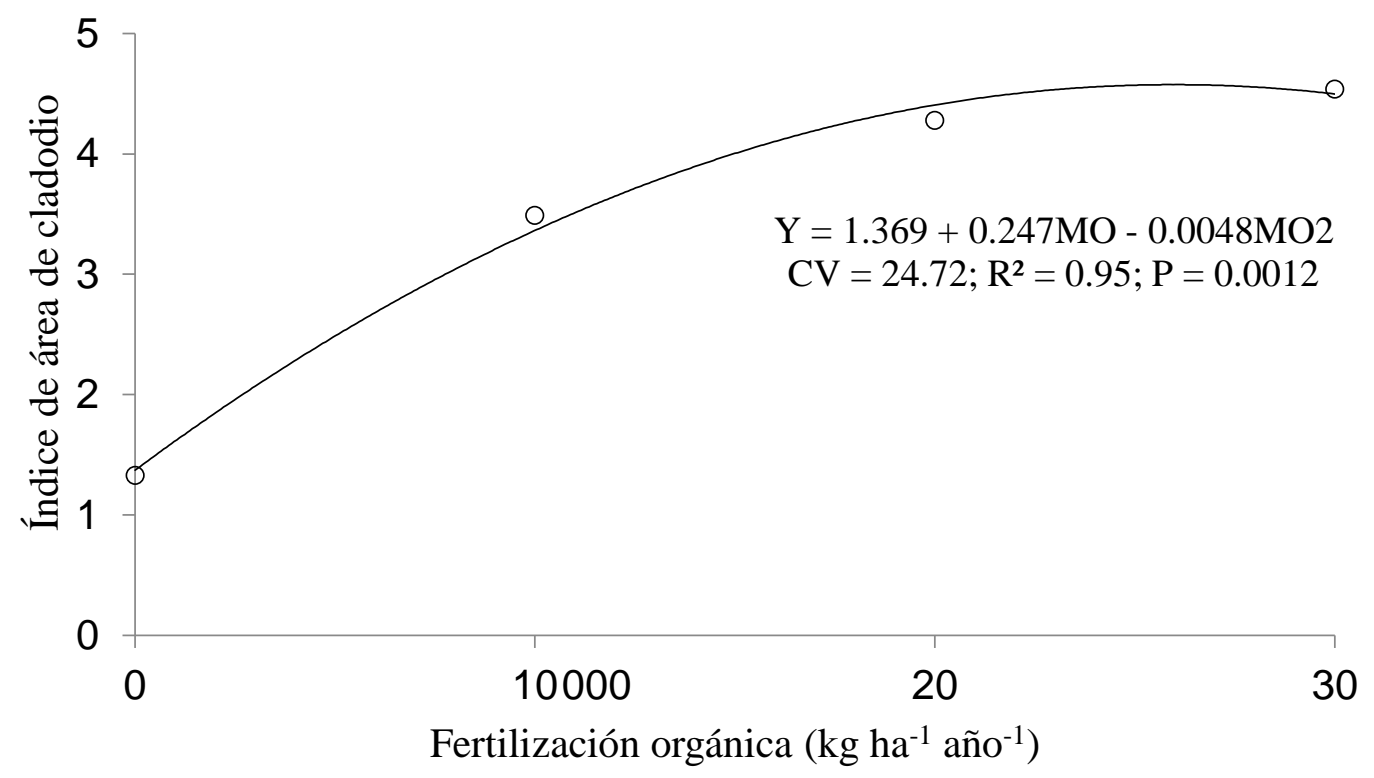

La frecuencia de corte tuvo efecto sobre el IAC $(P \leq 0.05)$. Las plantas con corte a dos años mostraron un IAC mayor (4.7) $(P \leq 0.05)$ que aquella de corte anual (2.2). Esto se atribuyó a que con el corte anual el área fotosintética residual fue menor que las plantas de corte bianual, 
un bajo IAC reduce la intercepción de luz y el crecimiento de la planta ${ }^{(27)}$. Respecto a la intensidad de corte, se reportó que la producción de materia seca en $O$. ficus-indica fue mayor cuando se conservaron los cladodios de orden secundario en el corte ${ }^{(28)}$. Lo anterior puede deberse a que las plantas con cladodios de nivel secundario tienen un mayor IAC que representa una mayor área fotosintética ${ }^{(29,30)}$.

En el Cuadro 2 se observa el coeficiente de correlación entre la productividad y las variables morfológicas, mostrando una alta correlación entre las variables evaluadas, por lo que la medición de variables morfológicas puede apoyar y ser un indicador de la productividad.

Cuadro 2: Coeficiente de correlación entre variables morfológicas y producción (t MS) de nopal forrajero (Nopalea cochenillifera Salm Dyck) cv. Miuda

\begin{tabular}{|c|c|c|c|c|c|}
\hline Variables & Producción $^{\ddagger}$ & IAC & Ancho & Longitud & Perímetro \\
\hline Producción $^{\ddagger}$ & & $0.94 * *$ & $0.95 * *$ & $0.82 * *$ & $0.91 * *$ \\
\hline IAC & & & $0.86^{* *}$ & $0.86 * *$ & $0.83 * *$ \\
\hline Ancho & & & & $0.81 * *$ & $0.95 * *$ \\
\hline Longitud & & & & & $0.88 * *$ \\
\hline Perímetro & & & & & \\
\hline
\end{tabular}

Se concluye que la fertilización orgánica con estiércol bovino incrementa el ancho, longitud e índice de área de cladodio (IAC), mientras que la fertilización mineral con urea tuvo bajo impacto en dichas características. El índice de área de cladodio (IAC) es mayor cuando el corte es bianual en este tipo de cultivo. Existe una alta correlación entre las variables morfológicas y de producción, debido a ello la importancia del estudio de variables morfológicas en el cultivo.

\section{Agradecimientos}

Este trabajo fue parte de la estancia de investigación del primer autor, bajo la supervisión del Dr. Luis Alberto Miranda Romero y la Dra. Mercia Virginia Ferreira dos Santos. Los autores agradecen el soporte financiero recibido del CONACYT, Universidad Federal Rural de Pernambuco y Universidad Autónoma Chapingo. 


\section{Literatura citada:}

1. Ministério da Integração Nacional. Nova Delimitação do Semi-Árido Brasileiro. Secretaria de Políticas de Desenvolvimento Regional. Brasília, D.F. Brasil. 2005.

2. Silva VPR, Pereira ERR, Almeida RSR. Estudo da variabilidade anual e intra-anual da precipitação na região nordeste do Brasil. Rev Bras Metereo 2012;27(2):163-172.

3. Fuentes-Rodríguez J. Feeding prickly pear cactus to small ruminants in Northern Mexico. I. Goats. J Prof Assoc Cactus Dev 1997;2:23-25.

4. Luttge U. Ecophysiology of crassulacean acid metabolism (CAM). Ann Bot 2004;93:629652.

5. De Waal HO, Zeeman DC, Combrinck JW. Wet faeces produced by sheep fed dried spineless cactus pear cladodes in balanced diets. S Afr J Anim Sci 2006;36:10-13.

6. Galvão Jr. JGB, Silva JBA, Morais JHG, Lima RN. Palma forrageira na alimentação de ruminantes: cultivo e utilização. Acta Vet Bras 2014;8:78-85.

7. Vasconcelos AGV, Lira MA, Cavalcanti VALB, Santos MVF. Seleção de clones de palma forrageira resistentes à cochonilha do carmim (Dactylopius ceylonicus). Rev Bras Zootecn 2009;38:827-831.

8. Neves ALA, Pereira LGR, Santos RD, Voltolini TV, Araújo SA, Moraes SA, Aragão ASL, Costa CTF. Plantio e uso da palma forrageira na alimentação de bovinos leiteiros no Semiárido brasileiro. $1^{a}$ ed. Embrapa Gado de Leite, Juiz de Fora M. G. Comunicado Técnico 62. 2010.

9. Batista AM, Mustafa AF, McAllister T, Wang Y, Soita H, McKinnon JJ. Effect of variety on chemical composition, in situ nutrient disappearance and in vitro gas production of spineless cacti. J Sci Food Agric 2003;83:440-445.

10. Dubeux Jr. JCB, Araújo Filho JT, Santos MVF, Lira MA, Santos DC, Pessoa RAS. Adubação mineral no crescimento e composição mineral da palma forrageira clone IPA20. Rev Bras Ciênc Agrár 2010;5:129-135.

11. Santos DC, Farias I, Lira MA, Santos MVF, Arruda GP, Coelho RSB, Dias FM, Melo JN. Manejo e utilização da palma forrageira (Opuntia e Nopalea) em Pernambuco. Empresa Pernambucana de Pesquisa Agropecuária (IPA). Documentos 30. Recife. Brasil. 2006.

12. Neder DG, Costa FR, Edvan RL, Filho LTS. Correlations and path analysis of morphological and yield traits of cactus pear accessions. Crop Breed Appl Biotechnol 2013;13:203-207. 
13. Brito CILV, Ffolliot PF, Peixoto PSA. Uma classificação morfo-estructural para descrição e avaliação da biomassa da vegetação da caatinga. Rev Caatinga 2008;21(2):204-213.

14. EMBRAPA (Empresa Brasileira de Pesquisa Agropecuária) Centro Nacional de Pesquisa de Solos. Sistema brasileiro de classificação de solos. 3ed. Rio de Janeiro: Embrapa Solos. 2013.

15. EMBRAPA (Empresa Brasileira de Pesquisa Agropecuária). Manual de Métodos de Análise de Solo. Centro Nacional de Pesquisa de Solos. 2a ed. Rio de Janeiro, Brasil. 1997.

16. AOAC (Association of Official Agricultural Chemists). Official Methods of Analysis. 15th. ed. Association of Official Agricultural Chemists, Washington, DC. 1990.

17. Nobel PS, Bobich EG. Environmental biology. In: Nobel PS editor. Cacti: Biology and Uses. University of California Press, California, USA. 2002.

18. Souza TC. Sistemas de cultivo para palma forrajeira cv Miúda (Nopalea cochenillifera Salm Dyck) [tesis doctorado]. Brasil, PR: Universidade Federal Rural de Pernambuco; 2015.

19. SAS (Statistical Analysis System). SAS/STAT User's Guide (Release 9.3). Cary, NC, USA. SAS Inst. Inc. 2012.

20. Cunha DNFV, Gomes ES, Martuscello JA, Amorim PL, Silva RC, Ferreira PS. Morfometria e acúmulo de biomassa em palma forrageira sob doses de nitrogênio. Rev Bras Saúde Produç Anim 2012;13:1156-1165.

21. González CL. Potential of fertilization to improve nutritive value of prickly pear cactus (Opuntia lindheimeri Engelm.) J Arid Environ 1989;16:87-94.

22. Julca-Otiniano A, Meneses-Florián L, Blas-Sevillano R, Bello-Amez S. La materia orgánica, importancia y experiencias de su uso en la agricultura. IDESIA 2006;24:4961.

23. Trejo-Escareño HI, Salazar-Sosa E, López-Martínez JD, Vázquez-Vázquez C. Respuesta del nopal forrajero (Opuntia fícus-indica L.) a la aplicación de estiércol bovino solarizado. AGROFAZ 2014;14:87-95.

24. Donato PER., Pires AJV, Donato SLR, Bonomo P, Silva JA, Aquino AA. Morfometria e rendimento da palma forrageira 'Gigante' sob diferentes espaçamentos e doses de adubação orgânica. Rev Bras Ciênc Agrár 2014;9:151-158.

25. Nobel PS. Ecophysiology of Opuntia ficus-indica. In: Mondragon-Jacobo C, PerezGonzalez S editors. Cactus (Optunia spp.) as forage. FAO Plant Protection and Production Paper 169, Rome, Italy. 2002. 
26. García CV, Varnero MT, Espinoza M. Efecto de bioabono sobre el área fotosintéticamente activa, producción de cladodios y eficiencia de recuperación de $\mathrm{N}$ en un cultivo de tuna (Opuntia ficus-indica L.) en el primer año post-plantación. J Prof Assoc Cactus Dev 2001;4:93-104.

27. Dubeux JCB, Ben Salem H, Nefzaoui A. Producción y utilización de nopal forrajero en la nutrición animal. En: Inglese P, Mondragon JC, Nefzaoui A, Sáenz C. Ecología del cultivo, manejo y usos del nopal. FAO, Roma, Italia. 2018.

28. Alves RN, Farias I, Menezes C, Simões R, Lira MA, Santos DC. Produção de forragem pela palma após 19 anos sob diferentes intensidades de corte e espaçamentos. Rev Caatinga 2007;20:38-44.

29. Farias I, Lira MA, Santos DC, Filho JJT, Santos MVF, Fernandes APM, Santos VF. Manejo de colheita e espaçamento da palma-forrageira, em consórcio com sorgo granífero, no agreste de Pernambuco. Pesqui Agropecu Bras 2000;35:341-347.

30. Lira MA, Santos MVF, Dubeux Jr. JCB, Mello ACL. Sistema de produção de forragem: alternativas para sustentabilidade da pecuária. Rev Bras Zootec 2006;35:491-511. 